\title{
IDOSOS E TECNOLOGIAS: UMA PESQUISA
} BIBLIOGRÁFICA

\author{
Elimara Oliveira Costa ${ }^{2}$ \\ Amelia Carla Sobrinho Bifano ${ }^{3}$
}

resumo

O presente artigo trata de uma pesquisa bibliográfica que teve por objetivo mapear a produção acadêmica relacionada ao uso de tecnologias no cotidiano por idosos. Foi utilizado como método de investigação o Estado da Arte. Para a coleta de dados, foram selecionadas as bases de dados Scielo, Google Acadêmico e Periódicos Capes. Como critérios para seleção, foram consideradas as pesquisas que apresentassem no título as palavras "idoso" e "tecnologia" ou que tratasse do uso de um produto tecnológico específico pelo longevo, utilizando como descritores: "impacto do uso de tecnologias por idosos". No período compreendido pelo estudo apenas um autor fez parte de um volume maior de produções. Sobre a área de formação dos pesquisadores, destacou-se a Educação. As pesquisas qualitativas e as quanti-quali

\footnotetext{
1 Agradecimentos ao CNPq, pela bolsa de Iniciação Científica e à FAPEMIG pelofinanciamento do projeto.

2 Graduada em Economia Doméstica. Mestranda em Economia Doméstica pela Universidade Federal de Viçosa (UFV). E-mail: elimara.costa@ufv.br.

3 Graduada em Economia Doméstica. Doutora em Engenharia de Produção. Professora Adjunta da Universidade Federal de Viçosa (UFV), vinculada ao Departamento de Economia Doméstica.

E-mail: abifano@ufv.br.
} 
obtiveram maior frequência. Em termos de conteúdo, as publicações discorrem sobre o contato com computadores, próteses auditivas, eletrodomésticos, tecnologia educacional entre outras tecnologias. É importante levar em consideração que esta busca se baseou em apenas três bases de dados, podendo outros resultados serem obtidos caso haja uma ampliação da mesma e dos descritores.

palavras-chave

Estado da Arte. Produção Acadêmica. Idosos. Tecnologia.

\section{Introdução}

Este estudo trata do levantamento acerca do conhecimento produzido nos últimos dez anos, ou seja, no período de 2004 a 2014, sobre o uso de tecnologias na vida cotidiana por idosos no Brasil. Buscou-se identificar a frequência de produções por autor, suas respectivas áreas de formação, a metodologia utilizada e os conteúdos destas produções.

A palavra tecnologia (do grego $\tau \varepsilon \chi \nu \eta$ - "ofício" - e $\lambda$ o $\gamma\llcorner\alpha$ - "estudo") é compreendida como um termo que envolve o conhecimento técnico e científico e as ferramentas, processos e materiais criados e/ou utilizados a partir de tal conhecimento (BIFANO, 2016). Além de informação, lógica e coisas, a tecnologia é um empreendimento fundamentalmente humano: é o modo como os seres humanos vivem e desenvolvem suas diversas atividades em contextos sociais e históricos específicos, com interesses e intenções distintas (THEIS, 2009).

Como temática recente, pouco se conhece acerca dos estudos e das áreas de conhecimento que estão envolvidas com os estudos referentes ao impacto do uso de tecnologias por idosos. A ideia de um levantamento que possibilite a formação de um quadro geral a respeito do que se tem estudado acerca desta temática, quais áreas do conhecimento têm maior investimento, assim como quais os conteúdos abordados, se torna uma ferramenta útil.

De acordo com Affonso (2013), conforme recentes pesquisas realizadas pelo Instituto Brasileiro de Geografia e Estatística (IBGE), a expectativa de vida da população se eleva continuamente. A projeção é a de que os idosos no Brasil devam representar $26,7 \%$ da população $(58,4$ milhões de idosos para uma população de 218 milhões de pessoas) em 2060, numa proporção 3,6 vezes maior do que a representada pelo contingente de 15 milhões de pessoas com 60 anos ou mais de idade ( $8,6 \%$ da população brasileira) no ano de 2012. Devido 
a esse crescente aumento do número de idosos e ao simultâneo avanço das tecnologias e da eletrônica nos produtos tecnológicos envolvidos nas atividades cotidianas, os termos tecnologia e envelhecimento, de acordo com Raymundo (2013), necessitam ser averiguados em sua relação, principalmente porque a população idosa vivencia um processo de dificuldade no enfrentamento e na utilização destas tecnologias em seu cotidiano. Dentre os diversos motivos responsáveis por essa dificuldade, segundo Amaral Junior (2013), pode-se citar a falta de conhecimento sobre os requisitos técnicos do produto, como a dificuldade de interpretar as informações que o mesmo traz, a ausência de informações claras nos manuais de instruções, bem como a percepção negativa que o idoso tem de si mesmo, afetando sua autonomia.

De acordo com Amaral Junior (2013) a autonomia dos sujeitos está relacionada a um conjunto de fatores. Dentre eles a forma como os sujeitos se posicionam no mundo, como veem a si próprios e aos outros, assim como a maneira como constroem essas relações, visto que, a maneira como cada sujeito se percebe, reflete na sua forma de pensar e de agir, bem como na realização de suas atividades, sendo, portanto, responsáveis pelo sucesso e insucesso de diversas ações.

Entende-se que as pessoas idosas se percebem como incapazes de usar diferentes tipos de tecnologias. Tal percepção é originária de uma visão estereotipada na sociedade ocidental, onde a velhice é compreendida como o estágio da decadência e da improdutividade. Além do paradoxo de um crescente acesso dos idosos a diversas tecnologias por um lado, e um aumento da exclusão, por outro, destaca-se a divulgação destas tecnologias como aquelas que podem proporcionar aos idosos uma vida mais autônoma e menos dependente de outras pessoas na realização de atividades básicas do cotidiano, tanto em produtos tecnológicos de uso pessoal, quanto no ambiente domiciliar.

\section{Método}

Foi utilizado como método de pesquisa bibliográfica o Estado da Arte, que objetiva:

[...] mapear e discutir certa produção acadêmica em diferentes campos do conhecimento, tentando responder que aspectos e dimensões vêm sendo destacados e privilegiados em diferentes épocas e lugares, de que formas e em que condições têm sido produzidas certas dissertações de mestrado, teses de doutorado, publicações em periódicos e comunicações em anais de congressos e de seminários (FERREIRA, 2002, p. 258). 
Realizou-se a busca por publicações que contemplassem a temática do impacto do uso de tecnologias por idosos. As bases de referência de dados foram Google Acadêmico (https://scholar.google.com.br/), Scientific Electronic Library Online - Scielo (http://scielo.org/php/index.php) e no portal de Periódicos da Coordenação de Aperfeiçoamento de Pessoal de Nível Superior - Capes (http://periodicos.capes.gov.br/), combinando os descritores: impacto do uso de tecnologia por idosos, sem aspas, no intervalo de 2004 a 2014.

Na base de dados do Google Acadêmico foi selecionada a opção "Pesquisa avançada", usando como critério a opção "Tudo no título". Na base de dados da Scielo, selecionou-se a opção "pesquisa de artigos", no campo "palavras do título"; na base de dados Periódicos Capes, inicialmente selecionou-se a opção portal de periódicos, em seguida a opção buscar assunto. Foram encontradas um total de 15.740 produções. A partir desta primeira seleção, utilizou-se como critério para uma filtragem mais específica, a leitura dos títulos de cada produção e seleção daquelas em que ocorresse obrigatoriamente a presença dos termos "idoso" e "tecnologia", ou em que houvesse relação com o uso de uma tecnologia específica por um idoso. Dos iniciais 15.740, permaneceram 45 produções. Por fim, após a leitura dos resumos das 45 produções, foram selecionadas somente aquelas em cujo conteúdo ocorrera relato de experiência de idosos com uma tecnologia e/ou estudos acerca dos tipos de tecnologias aos quais os mesmos tinham acesso. Foram então selecionadas 25 produções, que compuseram este estudo.

Para o tratamento dos dados, procedeu-se à análise do conteúdo das produções selecionadas, seguindo a proposta por Bardin (2009), que a define como o conjunto de técnicas de análise das comunicações que fazem uso de procedimentos sistemáticos e objetivos de descrição do conteúdo das mensagens (SILVA et al., 2013). Foi utilizado o programa de análise lexical WordSmith Tools versão 6.0, ano 2012, para o tratamento dos dados. “O software consiste de um conjunto de diferentes programas com várias aplicações, que compreendem o pré-processamento, a organização de dados e a análise propriamente dita de corpora ou textos isolados" (SARDINHA, 2005, p. 184). Utilizou-se o título da produção e o objetivo geral apresentado pelos autores das publicações, na ferramenta Word List, que gerou uma listagem com as palavras mais recorrentes nos textos inseridos no programa. Desta listagem, foram selecionadas palavras/termos utilizando-se os seguintes critérios:

1. Frequência no texto;

2. Frequência no mesmo texto e em outros;

3. Palavra/termo relacionada à tecnologia/idosos. 
Percebeu-se, após o uso da ferramenta Word List, uma elevada frequência de palavras como estudo, presente, objetivo; esses termos não foram considerados neste artigo por se tratar da linguagem acadêmica que dá a forma ao texto. Utilizou-se em seguida, a ferramenta Concord para a localização dos textos nos quais houve a ocorrência da palavra/termo geradas pela Word List. Foram lidos os resumos listados pelo Concord onde os termos apareciam e, posteriormente os textos foram agrupados por similaridade de conteúdo.

A partir da leitura dos resumos, montou-se um banco de dados com as principais informações colhidas. Inicialmente buscou-se verificar os seguintes itens: título, primeiro autor, especificação e ano da publicação. Foram analisadas as publicações que trataram das temáticas: idoso e tecnologia, e impacto do uso da tecnologia como temática principal. Dessa forma, foram excluídos os trabalhos que mencionaram o assunto apenas como parte secundária. Após o levantamento das produções procedeu-se à leitura. Para compreensão do conteúdo dos estudos, foram lidos todos os artigos. Dissertações, teses, trabalhos de conclusão de curso e relatório de iniciação científica, por se tratarem de estudos de maior volume de conteúdo, selecionou-se para a leitura apenas os segmentos que apresentavam contribuição para este estudo. Dessa forma, foram lidos os resumos, a metodologia, resultados e conclusões.

\section{Resultados e Discussões}

Os resultados e suas discussões serão apresentados em forma de tópicos, especificando o ano de publicação das produções, as abordagens metodológicas utilizadas e o estudo do conteúdo das mesmas.

\subsection{Análise das produções quanto ao ano de publicação}

O Gráfico 1 apresenta a distribuição dos trabalhos publicados, de acordo com a frequência por ano de publicação. 


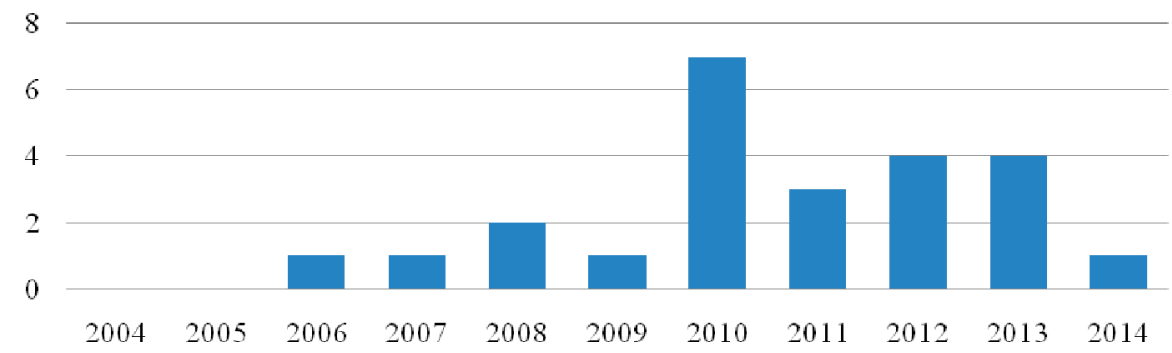

Fonte: Dados da pesquisa, 2017.

Conforme apresentado no Gráfico 1, pode-se observar que nos anos de 2004 e 2005 não ocorreram publicações que relacionassem idosos e tecnologias. Destacam-se os anos de 2010, 2012 e 2013 em que ocorreram seis e quatro publicações por ano respectivamente, seguidos dos anos de 2011 com três produções, 2008 e 2014 com duas produções e os anos de 2006, 2007 e 2009, com apenas uma produção em cada ano. Percebe-se que o investimento na temática ainda é muito limitado, o que corrobora com os estudos de Raymundo (2013) e Silva (2011), que destacam a falta de estudos relacionados ao uso de tecnologias. De acordo com Raymundo (2013), estes estudos estão concentrados em países considerados desenvolvidos e de primeiro mundo, como Europa e Estados Unidos, estados onde tanto a tecnologia e o acesso a elas estão mais avançados que no Brasil, como também são regiões em que a longevidade já é uma realidade; portanto, a problemática no uso de tecnologias por idosos já se apresenta.

\subsection{Análise das produções quanto aos tipos de pesquisas utilizadas}

No Quadro 1, estão apresentados os tipos de pesquisas utilizadas nas publicações, a quantificação destes tipos e as técnicas de coleta de dados de acordo com seu respectivo método. 
Quadro 1 - Tipos de pesquisas utilizadas nos estudos selecionados.

\begin{tabular}{|c|c|c|c|}
\hline Tipo de Pesquisa & $\mathrm{N}^{\circ}$ & $\begin{array}{c}\text { Técnicas de coleta de } \\
\text { dados }\end{array}$ & $\mathrm{N}^{\circ}$ \\
\hline \multirow{8}{*}{ Qualitativa } & \multirow{8}{*}{14} & Entrevista & 08 \\
\hline & & Questionário & 05 \\
\hline & & Pesquisa bibliográfica & 03 \\
\hline & & Relatos & 03 \\
\hline & & Pesquisa documental & $\mathrm{O} 2$ \\
\hline & & Diário de campo & 02 \\
\hline & & Depoimentos & 01 \\
\hline & & Observação participante & 01 \\
\hline \multicolumn{3}{|l|}{ Subtotal } & 25 \\
\hline \multirow{2}{*}{ Quantitativa } & \multirow{2}{*}{02} & Questionário & 01 \\
\hline & & Entrevista & 01 \\
\hline \multicolumn{3}{|l|}{ Subtotal } & 02 \\
\hline \multirow{6}{*}{ Quanti-qualitativa } & \multirow{6}{*}{09} & Questionário & 12 \\
\hline & & Escalas de avaliação & 05 \\
\hline & & Pesquisa documental & 02 \\
\hline & & Protocolos & 01 \\
\hline & & Cadernetas de setor & 01 \\
\hline & & Diário de campo & 01 \\
\hline \multicolumn{3}{|l|}{ Subtotal } & 22 \\
\hline
\end{tabular}

Fonte: Dados da pesquisa, 2017.

Quanto aos tipos de pesquisas utilizadas, conforme dados apresentados no Quadro 1, pode-se observar a ocorrência de uma variabilidade de métodos e técnicas em cada produção. Em um mesmo estudo, por exemplo, identificou-se o emprego de mais de cinco técnicas. Das 25 publicações, quatorze usaram a pesquisa qualitativa, dentre elas: História Oral Temática; Estudo Transversal; Estudo de Caso e Pesquisa Exploratória.

Quanto aos instrumentos aplicados para coleta de dados, quatro foram entrevistas; três recorreram ao questionário; duas optaram pelo relato de experiência; uma recorreu simultaneamente à entrevista e ao questionário; 
uma empregou conjuntamente entrevista, pesquisa bibliográfica e diário de campo; uma usou pesquisa bibliográfica, pesquisa documental, relatos e depoimentos de forma conjunta; uma recorreu por usar concomitantemente pesquisa bibliográfica, pesquisa documental e entrevista; uma recorreu à observação participante, diário de campo, entrevista e questionário no mesmo estudo; prevalecendo a entrevista como instrumento de coleta de dados nos trabalhos com pesquisas qualitativas. Nas duas produções que utilizaram a pesquisa quantitativa, os métodos utilizados foram, respectivamente, Estudo Descritivo, não Experimental do tipo Transversal e Estudo Transversal, Observacional Descritivo.

Para a coleta de dados, foram utilizados a entrevista e o questionário, respectivamente. Em nove publicações foi empregado o enfoque quantitativo-qualitativo. Sendo os tipos apresentados a Pesquisa Exploratória, Descritiva, do tipo Transversal; o Estudo Clínico e Experimental; o Estudo de Caráter Transversal; o Estudo Prospectivo, Exploratório, Descritivo e Transversal. Entre as técnicas de coleta de dados, duas publicações utilizaram o questionário; uma produção optou pela pesquisa documental; uma publicação usou concomitantemente questionário sociodemográfico auto respondido, questionário estruturado, escala de avaliação das atividades instrumentais da vida diária de Lawton e Brody; uma produção utilizou conjuntamente o questionário, protocolos de avaliação de capacidade funcional e qualidade de vida; uma recorreu conjuntamente a caderneta do setor, questionários de amostras, questionário multidimensional para estudos comunitários na população idosa, questionários semi-estruturados e diário de campo; uma produção usou no mesmo estudo questionário socioeconômico, escala de avaliação das atividade instrumentais da vida diária de Lawton e Brody, escala para avaliação das aceitações da tecnologia e questionário e por fim; uma publicação fez uso da escala de Mini Exame do Estado Mental (MMSE), de indicadores socioeconômicos e demográficos de gênero, da escolaridade, do estado civil e da renda familiar.

A justificativa que prevalecente nos quatorze estudos que utilizaram a pesquisa qualitativa foi a de que esta promove um contato mais aprofundado do pesquisador com seu objeto de estudo. Em relação aos métodos de coleta de dados, o questionário, a entrevista, a escala de avaliação das atividades instrumentais da vida diária de Lawton e Brody, escala de aceitação de tecnologias, baseadas nos princípios da Escala de Atitudes em relação ao uso de computador de autoria de Gilbert, Lee-Kelly, Barton (2003) - esta escala tem por objetivo avaliar a aceitação de tecnologias por idosos -, a escala de Mini Exame do Estado Mental (MMSE), foram os procedimentos empregados. O 
uso do questionário promoveu a caracterização da amostra, além de facilitar o processo de análise estatística (CARLETO, 2013; ESTEVES, 2014; PASQUALOTTI, 2008). No entendimento dos pesquisadores que optaram pela entrevista, esta permite flexibilidade e oportunidade de se avaliar atitudes e condutas, além da observação do comportamento do entrevistado. A utilização das escalas foi justificada por ser um instrumento eficiente na avaliação de tendências de comportamentos.

\subsection{Análise do conteúdo das publicações}

As publicações foram organizadas a partir de seus conteúdos de maior significação, em quatro agrupamentos, cujas temáticas estão apresentadas a seguir:

1. Tecnologias de Informação e Comunicação (TIC);

2. Tecnologias Voltadas à Saúde do Idoso;

3. Aceitação de Tecnologias pelos Idosos;

4. Tecnologias em Ambientes Domésticos.

O Quadro 2 mostra o agrupamento das publicações dentro das quatros áreas temáticas, com discriminação de títulos, autores, especificações e ano das publicações. 
Quadro 2 - Título, autores, especificação e ano da publicação.

\begin{tabular}{|c|c|c|c|c|}
\hline \multirow{8}{*}{ 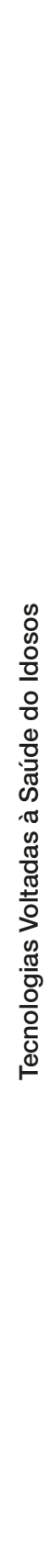 } & Titulo & Autor(es) & $\begin{array}{l}\text { Especifi- } \\
\text { cação da } \\
\text { Publicação }\end{array}$ & Ano \\
\hline & $\begin{array}{l}\text { 1. Deficiência auditiva em idosos: relatos } \\
\text { orais sobre o impactoproporcionado pelo } \\
\text { uso do Aparelho de Amplificação Sonora }\end{array}$ & LUTFI, A. & Dissertação & 2006 \\
\hline & $\begin{array}{l}\text { 2. O uso de próteses auditivas por } \\
\text { idosos: os repertórios interpretativos } \\
\text { utilizados nessa decisão }\end{array}$ & RIBEIRO, L. M. & Dissertação & 2008 \\
\hline & $\begin{array}{l}\text { 3. Processo de adaptação de aparelho } \\
\text { de amplificação sonora individual: } \\
\text { elaboração de um DVD para auxiliar a } \\
\text { orientação a indivíduos idosos }\end{array}$ & $\begin{array}{l}\text { CAMPOS, } \\
\text { K.; OLIVEIRA, } \\
\text { J. R. M. de; } \\
\text { BLASCA, W. Q. }\end{array}$ & Art. Científico & 2010 \\
\hline & $\begin{array}{l}\text { 4. A expectativa como fator de influên- } \\
\text { cia no sucesso com o uso de próteses } \\
\text { auditivas, em indivíduos idosos }\end{array}$ & $\begin{array}{l}\text { SCHUSTER, L. } \\
\text { C.; COSTA, M. } \\
\text { J.; MENE- } \\
\text { GOTTO, I. H. }\end{array}$ & Art. Científico & 2012 \\
\hline & $\begin{array}{l}\text { 5. Avaliação da tecnologia das relações } \\
\text { de cuidado nos serviços em saúde: per- } \\
\text { cepção dos idosos inseridos na Estratégia } \\
\text { Saúde da Família em Bambuí, Brasil }\end{array}$ & $\begin{array}{l}\text { SANTOS, W. } \\
\text { J.; GIACOMIN, } \\
\text { K. C.; FIRMO, } \\
\text { J. O. A. }\end{array}$ & Art. Científico & 2014 \\
\hline & $\begin{array}{l}\text { 6. Tecnologia educacional inovadora } \\
\text { para o empoderamento junto a idosos } \\
\text { com diabetes mellitus }\end{array}$ & $\begin{array}{l}\text { HAMMERSCH- } \\
\text { MIDT, K. S. de } \\
\text { A. LENARDT, } \\
\text { M. H. }\end{array}$ & Art. Científico & 2010 \\
\hline & $\begin{array}{l}\text { 7. Compreendendo o idoso usuário de } \\
\text { próteses auditivas }\end{array}$ & $\begin{array}{l}\text { PAIVA, K. M.; } \\
\text { MACIEL, P. M. } \\
\text { A.; CINTRA, } \\
\text { L. G. }\end{array}$ & Art. Científico & 2011 \\
\hline
\end{tabular}




\begin{tabular}{|c|c|c|c|c|}
\hline & Titulo & Autor(es) & $\begin{array}{l}\text { Especifi- } \\
\text { cação da } \\
\text { Publicação }\end{array}$ & Ano \\
\hline & $\begin{array}{l}\text { 8. O impacto sociocultural do uso } \\
\text { da internet em um grupo de pessoas } \\
\text { idosas }\end{array}$ & REIS, A. A. & Dissertação & 2012 \\
\hline & $\begin{array}{l}\text { 9. Relações intergeracionais de idosos } \\
\text { mediadas pelas tecnologias de informa- } \\
\text { ção e comunicação }\end{array}$ & $\begin{array}{l}\text { CARLETO, D. } \\
\text { G. de S. }\end{array}$ & Dissertação & 2013 \\
\hline & $\begin{array}{l}\text { 10. Uso da internet pelo consumidor } \\
\text { da terceira idade: influências do risco } \\
\text { percebido e impacto na intenção de compra } \\
\text { on-line }\end{array}$ & $\begin{array}{l}\text { ESTEVES, } \\
\text { P. S. }\end{array}$ & Tese & 2014 \\
\hline & $\begin{array}{l}\text { 11. Ambientes de aprendizagem: signifi- } \\
\text { cado na vida de idosos frequentadores de } \\
\text { oficinas de informática }\end{array}$ & $\begin{array}{l}\text { SILVEIRA, M. } \\
\text { M. et al. }\end{array}$ & Art. Científico & 2011 \\
\hline 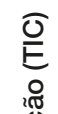 & $\begin{array}{l}\text { 12. Comunicação, tecnologia e envelhe- } \\
\text { cimento: significação da interação na era } \\
\text { da informação }\end{array}$ & $\begin{array}{l}\text { PASQUA- } \\
\text { LOTTI, A. }\end{array}$ & Tese & 2008 \\
\hline 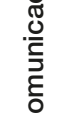 & $\begin{array}{l}\text { 13. Repositório digital como ambiente } \\
\text { de inclusão digital e social para usuá- } \\
\text { rios idosos }\end{array}$ & $\begin{array}{l}\text { VECHIATO, } \\
\text { F. L. }\end{array}$ & Dissertação & 2010 \\
\hline $\begin{array}{l}0 \\
\stackrel{0}{0} \\
2\end{array}$ & $\begin{array}{l}\text { 14. A relação do idoso com as novas } \\
\text { tecnologias: análise de mídia eletrônica }\end{array}$ & $\begin{array}{l}\text { PIOLA, B. C. } \\
\text { T.; SEI, M. N. }\end{array}$ & IC & 2009 \\
\hline 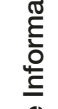 & $\begin{array}{l}\text { 15. Mudanças sociais e tecnológicas e } \\
\text { suas repercussões na vida das pessoas } \\
\text { idosas }\end{array}$ & $\begin{array}{l}\text { CARVALHO, } \\
\text { F.A. }\end{array}$ & TCC & 2010 \\
\hline 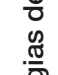 & $\begin{array}{l}\text { 16. Percepção do idoso em relação à } \\
\text { internet }\end{array}$ & $\begin{array}{l}\text { VERONA, S. } \\
\text { M. et al. }\end{array}$ & Art. Científico & 2010 \\
\hline 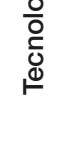 & $\begin{array}{l}\text { 17. As tecnologias de informação e } \\
\text { comunicação na vida de idosos com } \\
\text { sintomas de depressão: significado, } \\
\text { experiências e relacionamentos }\end{array}$ & $\begin{array}{l}\text { PASQUA- } \\
\text { LOTTI, A.; } \\
\text { BARONE, D. } \\
\text { A. C.; DOLL, J. }\end{array}$ & Art. Científico & 2007 \\
\hline & $\begin{array}{l}\text { 18. Reflexões acerca das contribuições } \\
\text { das tecnologias no desenvolvimento } \\
\text { de atividades, relações e identidades } \\
\text { sociais de idosos }\end{array}$ & $\begin{array}{l}\text { ELY, J. C.; } \\
\text { PASQUA- } \\
\text { LOTTI, A. }\end{array}$ & Art. Científico & 2010 \\
\hline & $\begin{array}{l}\text { 19. A inclusão dos idosos no mundo } \\
\text { digital através das novas tecnologias da } \\
\text { informação e comunicação (NTICs) }\end{array}$ & $\begin{array}{l}\text { SÁ, M. E. G.; } \\
\text { ALMEIDA, V. L. }\end{array}$ & Art. Científico & 2012 \\
\hline & $\begin{array}{l}\text { 20. Transformações societárias, envelhe- } \\
\text { cimento e novas tecnologias: os impactos } \\
\text { da informática na vida dos idosos }\end{array}$ & $\begin{array}{l}\text { EVANGELISTA, } \\
\text { K. Z.S. }\end{array}$ & TCC & 2010 \\
\hline
\end{tabular}


continuação

\begin{tabular}{|c|c|c|c|c|}
\hline & Titulo & Autor(es) & $\begin{array}{l}\text { Especifi- } \\
\text { cação da } \\
\text { Publicação }\end{array}$ & Ano \\
\hline ○े & $\begin{array}{l}\text { 21. Uso das novas tecnologias da } \\
\text { informação e comunicação entre idosos } \\
\text { frequentadores da UnATi/Uem: perfil, } \\
\text { motivações, interesses e dificuldades }\end{array}$ & $\begin{array}{l}\text { LOLLI, M. C. } \\
\text { G. S. et al. }\end{array}$ & Art. Científico & 2013 \\
\hline 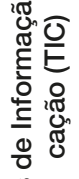 & $\begin{array}{l}\text { 22. Reflexões sobre aproximação de } \\
\text { idosos a tecnologias de informação e } \\
\text { comunicação a partir dos arquétipos } \\
\text { Senex* e Puer }\end{array}$ & $\begin{array}{l}\text { KATZENS- } \\
\text { TEIN, T. V.; } \\
\text { SCHWARTZ, } \\
\text { G.; MORGANI, } \\
\text { M. H. de A. }\end{array}$ & Art. Científico & 2012 \\
\hline 흥 & $\begin{array}{l}\text { 23. Idosos em rede: a construção de } \\
\text { novas práticas culturais mediadas por } \\
\text { tecnologias }\end{array}$ & $\begin{array}{l}\text { OLIVEIRA, K. } \\
\text { L. G. }\end{array}$ & Dissertação & 2013 \\
\hline 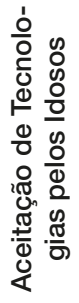 & 24. Aceitação de tecnologias por idosos & $\begin{array}{l}\text { RAYMUNDO, } \\
\text { T. M. }\end{array}$ & Dissertação & 2013 \\
\hline 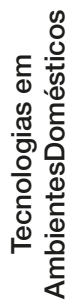 & $\begin{array}{l}\text { 25. Uso de aparelhos eletrônicos por } \\
\text { idosos em ambientes domésticos }\end{array}$ & SILVA, D. O. & Dissertação & 2011 \\
\hline
\end{tabular}

Fonte: Dados da pesquisa, 2017.

Conforme apresentado no Quadro 2, os conteúdos com maior frequência de publicações é o agrupamento denominado "Tecnologias de Informação e Comunicação", com dezesseis produções, seguido do "Tecnologias Voltadas à Saúde do Idosos", com sete estudos. Os dois outros trabalhos que obtiveram uma ocorrência foram "Aceitação de Tecnologias pelos Idosos" e "Tecnologias em Ambientes Domésticos", o que permitiu constatar que os estudos relacionados 
a estas temáticas são ainda insipientes quando comparadas com as publicações sobre idosos e tecnologias de informação por exemplo.

Com relação à especificação, das vinte e cinco produções, treze tratam-se de artigos científicos, oito de dissertações de mestrado, duas de teses de doutorado, dois de trabalhos de conclusão de curso e uma publicação trata-se de um relatório de iniciação científica. No que diz respeito à autoria, no período compreendido pela pesquisa, que foi de 2004 a 2014, Pasqualotti fez parte de quatro produções, não sendo observado o investimento maior de demais autores na temática abordada neste estudo.

Em termos da formação acadêmica, os autores de cinco publicações são da Educação, três da Fonoaudiologia, três da Enfermagem, três da Bioengenharia, dois da Psicologia, dois da Gerontologia, dois do Serviço Social e dois da Ciência da Comunicação. As demais publicações, cada uma delas com autores de formação em uma área de conhecimento, são respectivamente: Saúde Pública, Saúde Coletiva, Filosofia, Enfermagem, Administração, Ciência da Informação, Comunicação Social, Sistema de Informação, e Odontologia. Dentre as 25 publicações, uma não especifica a área de formação dos autores.

As produções que tratam de Tecnologias de Informação e Comunicação (TICs) abordam os temas: internet; informática; inclusão digital; relações intergeracionais mediadas pelas TICs; significaçõesdas TICs para os idosos; relação do idoso com as TICs; repercussões das TICs na vida do idoso; aproximação do idoso com a TIC e o perfil do idoso que utiliza a Tecnologia de Informação e Comunicação (TIC). Dos estudos agrupados nessa temática, sete (REIS, 2012; ESTEVES, 2014; VERONA et al.; 2006; SÁ; ALMEIDA, 2012; LOLLI et al., 2013; KATZENSTEIN; SCHWARTZ; MORGANI, 2012; OLIVEIRA, 2013) estudaram a interação do idoso com a internet. Os autores discutem como era o contato do idoso, bem como o que faziam na internet e quais sites eram acessados. Estes estudos demonstraram que, ao contrário do que circula em termos de senso comum, os idosos utilizam o computador e a internet para a realização de diversas tarefas em seu cotidiano, tais como contato com familiares distantes, compra on-line, atividades de lazer, acesso a informações sobre política, educação, economia entre outros. A alfabetização em informática foi abordada por três estudos (SILVEIRA et al., 2011; VECHIATO, 2010; CARVALHO, 2010).

Os estudos tratam dos motivos pelos quais os idosos decidiram aprender a usar o computador, as mudanças de comportamento a partir do uso da informática, o uso do computador como alternativa para lidar com doenças como depressão, além de lidar com a solidão. Para Tezza e Bonia (2011), a interação entre idosos e informática está relacionada principalmente ao comportamento, compartilhamento de informações e aprendizagem; e estes aspectos estão 
intimamente ligados ao contexto sociocultural onde estes indivíduos vivem e trocam experiências. Silveira et al. (2011), constataram que a informática juntamente com outras tecnologias são ferramentas importantes, cada vez mais presentes no cotidiano das pessoas. Ambos os artigos destacam a não familiaridade com a linguagem midiática, o que poderá sujeitar os indivíduos a processos de exclusão social. De acordo Kim (2008 apud VIEIRA, 2011), o uso de computador pelos idosos pode ser movido pelas seguintes motivações: acesso à internet de forma a realizar variadas tarefas básicas do seu cotidiano e manutenção de relações com outros indivíduos.

Os estudos acerca das tecnologias relacionadas ao cuidado da saúde do idoso trataram do impacto do uso de aparelhos auditivos e dos repertórios utilizados na decisão de uso da mesma; das relações de cuidado nos serviços em saúde e da questão do empoderamento como tecnologia educacional para o cuidado de si, em idosos com diabetes mellitus. Esta foi a segunda temática mais abordada, com sete ocorrências de estudos relacionados ao uso de aparelhos auditivos com cinco trabalhos (LUTFI, 2006; RIBEIRO, 2008; CAMPOS; OLIVEIRA; BLASCA, 2010; SCHUTER; COSTA; MENEGOTTO, 2011; PAIVA; MACIEL; CINTRA, 2011). Ao estudar os repertórios que levaram aos idosos a aceitarem ou se negarem a utilizar os aparelhos auditivos, destacaram-se como positivos a questão do respeito da família e dos amigos, que, segundo os idosos, retorna quando estão com os aparelhos de amplificação sonora; o incômodo da repetição; a busca da prevenção e autocuidado; a prótese como um recurso tecnológico benéfico; o reconhecimento das limitações da prótese; e a virtude da resignação. Os repertórios negativos mais utilizados foram o uso de estratégias alternativas; o desconforto maior que o benefício; o insucesso do outro; a relativização da necessidade e a transitoriedade da decisão.

No caso das tecnologias e educação, as autoras Hammerschmidt e Lenardt (2010) discutiram o empoderamento a partir do uso de uma tecnologia educacional em um estudo com um grupo de idosos com diabetes mellitus. O estudo de Santos, Giacomin e Firmo (2014) objetivou compreender a percepção dos idosos sobre a capacidade e efetividade dos atos em saúde produzidos nas relações de cuidado contextualizadas na Estratégia Saúde da Família (ESF). O estudo sobre a aceitação de tecnologias por idosos, de Raymundo (2013) apresentou a discussão sobre questões de como a população idosa lida com os desafios da tecnologia, assim como quais variáveis influenciaram no uso e na aceitação das mesmas em seu cotidiano. De acordo com Deci e Ryan (2000 apud RAYMUNDO, 2013) a aceitação ou não de uma tecnologia está relacionada a fatores intrínsecos e extrínsecos ao sujeito. Sendo que as variáveis intrínsecas incluem capacidade funcional, comportamento, competência, percepção da utilidade, 
prazer, dificuldades e facilidades encontradas nos recursos tecnológicos. Por sua vez, os fatores extrínsecos envolvem características sócio demográficas dos sujeitos, gênero, idade e ocupação.

Já o estudo de Silva (2011), sobre o uso de aparelhos eletrônicos por idosos em ambientes domésticos, procurou descrever quais aparelhos eletrônicos os idosos possuíam em seus lares, como se deu a utilização dos mesmos, pelos idosos nesses ambientes, bem como, os relatos de dificuldades no uso. Para Bouma (2010 apud RAYMUNDO, 2013) quanto maior o entendimento acerca dos fatores que exercem influências sobre os comportamentos e atitudes dos idosos, tanto melhor será a capacidade de prever suas reais necessidades e ambições, com maiores chances de êxito, além de promover o suporte ideal para melhoria de sua qualidade de vida.

\section{Considerações Finais}

Os resultados encontrados demonstram que no período compreendido pela pesquisa, ocorreu reduzido número de publicações nas diversas áreas de conhecimento relacionadas à temática do impacto do uso de tecnologias no cotidiano de idosos. Em relação ao número de publicações por autor no período compreendido pela pesquisa, apenas Pasqualotti publicou mais de um trabalho, fazendo parte de quatro estudos, não sendo observado um investimento maior de demais autores na temática abordada.

A formação acadêmica dos autores apresenta uma diversidade de áreas de conhecimento. Principalmente nas disciplinas relacionadas às biológicas enfermagem, fisioterapia, fonoaudiologia, enfermagem, saúde pública, saúde coletiva. A educação se destacou entre as demais áreas.

Dentre as tecnologias mais utilizadas pelos idosos, destacaram-se aquelas pertencentes ao grupo das tecnologias de informação e comunicação (TICs), mais especificamente o uso de computadores. Isso aponta maior interesse em estudar a interação dos idosos com as tecnologias de informação e comunicação em relação às demais com as quais convivem cotidianamente.

Em relação ao tipo de pesquisa, constatou-se que as qualitativas e as quanti-qualitativas, foram adotadas com maior frequência. As justificativas apresentadas foram que as técnicas de métodos quantitativos perdem em essência, as particularidades vivenciadas pelos sujeitos. Já as metodologias que englobam aspectos qualitativos permitem o contato maior entre pesquisador e sujeito estudado, portanto, um conhecimento mais aprofundado de suas realidades. O questionário apresentou maior ocorrência entre as técnicas utilizadas para 
coleta dos dados, com a justificativa de promover a caracterização da amostra e facilitar o processo de análise estatística na opinião.

Embora haja limitação explicitada, verificar o estado em que se encontra a produção relativa à temática pode ser útil e interessante aos futuros pesquisadores nesta área. É importante levar em consideração que esta pesquisa se baseou em apenas três bases de dados, podendo outros resultados serem encontrados caso haja uma ampliação das mesmas e dos descritores.

A relação exitosa entre envelhecimento e tecnologia torna-se importante no sentido da garantia acesso, de inclusão social e de ampliação da autonomia das pessoas de idade avançada, uma vez que as mudanças sociais e tecnológicas interferem diretamente em diversos aspectos da vida cotidiana dessa parcela da população.

\author{
ELDERLY AND TECHNOLOGIES: A \\ BIBLIOGRAPHICAL RESEARCH
}

\title{
abstract
}

This article is a bibliographical research whose objective was to map the academic production related to the use of technologies in the elderly's daily life. The State of Art was used as investigation method. For data collection, we selected Scielo, Google Scholar and Periodical Capes databases. As criteria for selection, we considered the studies that presented the words "elderly" and "technology" in the title or that dealt with the use of a specific technological product by the long-term, using as descriptors: "impact of the use of technologies by the elderly". In the period covered by the study, only one author was part of a larger volume of productions. Regarding the area of training of researchers, Education was highlighted. Qualitative research and quanti-quali were more frequent. In terms of content, the publications talk about the contact with computers, hearing aids, appliances, educational technology among other technologies. It is important to take into account that this search was based on only three databases, and other results can be obtained if there is an extension of the search and the descriptors.

keywords

State of the Art. Academic Works. Elder. Technology. 
AFFONSO, Julia. Proporção de idosos no Brasil deve ser três vezes maior em 2060, diz IBGE. UOL, Rio de Janeiro, 2013. Disponível em: <http://noticias.uol.com.br/cotidiano/ ultimas-noticias/2013/08/29/idosos-devem-ser-267-da-populacao-brasileira-em-2060-diz-ibge.htm>. Acesso em: 1 dez. 2014.

AMARAL JUNIOR, José Carlos do. Estudo da interação idoso e tecnologia no universo doméstico e sua relação com a autonomia. 2013. 154 f. Dissertação (Mestrado em Economia Doméstica)-Universidade Federal de Viçosa, Viçosa, MG, 2013.

BARDIN, Laurence. Análise de conteúdo: Laurence Bardin. Tradução Luís Antero Reto e Augusto Pinheiro. Lisboa: Edições 70, 2009. 281 p.

BIFANO, Amélia Carla Sobrinho. Notas de Aula. Viçosa, MG: Universidade Federal de Viçosa, 2016.

Uso cotidiano de produtos no âmbito doméstico: interface empresa e economia familiar. Oikos: Revista Brasileira de Economia Doméstica, Viçosa, MG, v. 26, n. 1, p. 174-204, 2015.

CAMPOS, Karis de; OLIVEIRA, Jerusa Roberta Massola de; BLASCA, Wanderléia Quinhoneiro. Processo de adaptação de aparelho de amplificação sonora individual: elaboração de um DVD para auxiliar a orientação a indivíduos idosos. Revista da Sociedade Brasileira de Fonoaudiologia, São Paulo, v. 15, n. 1, p. 19-25, jan. 2010.

CARLETO, Daniel Gustavo de Sousa. Relações intergeracionais de idosos mediadas pelas tecnologias de informação e comunicação. 2013. 79 f. Dissertação (Mestrado em Bioengenharia)-Universidade de São Paulo, São Carlos, SP, 2013.

CARVALHO, Fernanda Alves. Mudanças sociais e tecnológicas e suas repercussões na vida das pessoas idosas. 2010. 99 f. Trabalho de Conclusão de Curso (Graduação em Serviço Social)-Universidade Federal de Santa Catarina, Florianópolis, 2010.

ELY, Jaqueline Colombo; PASQUALOTTI, Adriano. Reflexões acerca das contribuições das tecnologias no desenvolvimento de atividades, relações e identidades sociais de idosos. Revista Intexto, Porto Alegre, v. 2, n. 23, p. 120-132, dez. 2010.

ESTEVES, Priscila Silva. Uso da internet pelo consumidor da terceira idade: influências do risco percebido e impacto na intenção de compra online. 2014. 271 f. Tese (Doutorado em Administração)-Universidade Federal do Rio Grande do Sul, Porto Alegre, 2014.

EVANGELISTA, Katiuscia Zanfonato dos Santos. Transformações societárias, envelhecimento e novas tecnologias: os impactos da informática na vida dos idosos. 2010 102 f. Trabalho de Conclusão de Curso (Graduação em Serviço Social)-Universidade Federal de Santa Catarina, Florianópolis, 2010.

FERREIRA, Norma Sandra de Almeida. As pesquisas denominadas "Estado da Arte". Educação e Sociedade, Campinas, v. 23, n. 79, p. 257-272, ago. 2002.

GILBERT, David; LEE-KELLEY, Liz; BARTON, Maya. Technophobia, gender influences and consumer decision-making for technology-related products. European Journal of Innovation Management, v. 6, n. 4, p. 253-263, 2003.

HAMMERSCHMIDT, Karina Silveira de Almeida; LENARDT, Maria Helena. Tecnologia educacional inovadora para o empoderamento junto a idosos com diabetes mellitus. Texto \& Contexto Enfermagem, Campinas, v. 19, n. 2, p. 358-365, jun. 2010.

KATZENSTEIN, Tamara Vivian; SCHWARTZ, Gilson; MORGANI, Maria Helena de Almeida. Reflexões sobre aproximação de idosos a tecnologias de informação e comunicação a partir dos arquétipos Senex e Puer. Kairós: Revista da Faculdade de Ciências Humanas e Saúde, Sorocaba, v. 15, n. 2, p. 203-219, jun. 2012. 
LOLLI, Maria Carolina Gobbi dos Santos et al. Uso das novas tecnologias da informação e comunicação entre idosos frequentadores da UnATi/Uem: perfil, motivações, interesses e dificuldades. Revista de Teorias e Práticas Educacionais, Maringá, v. 1, n. 1, p. 9-15, out./dez. 2013.

LUTFI, Aloha. Deficiência auditiva em idosos: relatos orais sobre impacto proporcionado pelo uso do aparelho de amplificação sonora. 2006. 67 f. Dissertação (Mestrado em Fonoaudiologia)-Pontifícia Universidade Católica de São Paulo, São Paulo, 2006.

OLIVEIRA, Karoline Leite Guedes de. Idosos em rede: a construção de novas práticas culturais mediadas por tecnologias. 2013. 159 f. Dissertação (Mestrado em Educação)-Universidade Federal do Rio Grande do Sul, Porto Alegre, 2013.

PAIVA, Karina Mary de; MACIEL, Paulete Maria Ambrósio; CINTRA, Letícia Guedes. Compreendendo o idoso usuário de próteses auditivas. Ciência \& Saúde Coletiva, Rio de Janeiro, v. 16, n. 6, p. 2927-2934, jun. 2011.

PASQUALOTTI, Adriano. Comunicação, tecnologia e envelhecimento: significação da interação na era da informação. 2008. 266 f. Tese (Doutorado em Informática da Educação)-Universidade Federal do Rio Grande do Sul, Porto Alegre, 2008.

PASQUALOTTI, Adriano; BARONE, Dante Augusto Couto; DOLL, Johannes. As tecnologias de informação e comunicação na vida de idosos com sintomas de depressão: significado, experiências e relacionamentos. Revista Novas Tecnologias na Educação, Porto Alegre, v. 5, n. 2, p. 1-8, 2007.

PIOLA, Bruno César Tozatti; SEl, Marisa Naomi. A relação do idoso com as novas tecnologias: análise de mídia eletrônica. 2009. 44 f. Relatório Final de Pesquisa (Iniciação Científica em Comunicação Social e Jornalismo)-Universidade Estadual Paulista, Bauru, SP, 2009

RAYMUNDO, Taiuani Marquine. Aceitação de tecnologias por idosos. 2013. 89 f. Dissertação (Mestrado em Bioengenharia)-Universidade de São Paulo, São Carlos, SP, 2013.

REIS, Adriana Araújo. O impacto sociocultural do uso da internet em um grupo de pessoas idosas. 2012. 127 f. Dissertação (Mestrado em Gerontologia)-Pontifícia Universidade Católica de São Paulo, São Paulo, SP, 2012.

RIBEIRO, Luciana Muniz. O uso de próteses auditivas por idosos: os repertórios interpretativos utilizados nessa decisão. 2008. 150 f. Dissertação (Mestrado em Psicologia)-Universidade Federal de Uberlândia, Uberlândia, MG, 2008.

SÁ, Maira Elisa Grassi de; ALMEIDA, Vera Lúcia de. A inclusão dos idosos no mundo digital através das novas tecnologias da informação e comunicação (NTICs). Conexões: Ciência e Tecnologia, Fortaleza, v. 6, n. 1, p. 1-14, mar. 2012.

SANTOS, Wagner Jorge dos; GIACOMIN, Karla Cristina; FIRMO, Josélia Oliveira Araújo. Avaliação da tecnologia das relações de cuidado nos serviços em saúde: percepção dos idosos inseridos na Estratégia Saúde da Família em Bambuí, Brasil. Ciência \& Saúde Coletiva, Rio de Janeiro, v. 19, n. 8, p. 3441-3450, ago. 2014.

SARDINHA, Tony Berber. A influência do tamanho do corpus de referência na obtenção de palavras-chave usando o programa computacional WordSmith Tools. The ESPecialist, São Paulo, v. 26, n. 2, p. 183-204, set. 2005.

SCHUSTER, Larissa Cristina; COSTA, Maristela Julio; MENEGOTTO, Isabela Hoffmeister. A expectativa como fator de influência no sucesso com o uso de próteses auditivas em indivíduos idosos. International Archives of Otorhinolaryngology, São Paulo, v. 16, n. 2, p. 201-208, jun. 2012

SILVA, Andressa Hennig et al. Análise de conteúdo: fazemos o que dizemos? Um levantamento de estudos que dizem adotar a técnica. In: ENCONTRO DE ENSINO E PESQUISA EM ADMINISTRAÇÃO E CONTABILIDADE, 4., 2013, Brasília, DF. Anais... Brasília, DF: ANPAD, 2013. 
SILVA, Danielly de Oliveira. Uso de aparelhos eletrônicos por idosos em ambientes domésticos. 2011. 75 f. Dissertação (Mestrado em Bioengenharia)-Universidade de São Paulo, São Carlos, SP, 2011.

SILVEIRA, Michele Marinho da et al. Ambientes de aprendizagem: significado na vida de idosos frequentadores de oficinas de informática. Revista Novas Tecnologias na Educação, Porto Alegre, v. 9, n. 1, p. 1-9, jul. 2011

TEZZA, Rafael; BONIA, Antonio Cezar. O idoso e a internet: uma etnografia sobre interação e aprendizagem. Perspectivas em Ciência da Informação, Belo Horizonte, v. 15, n. 1, p. 185-197, jan./abr. 2010.

THEIS, Ivo Marcos. Ciência \& tecnologia e desenvolvimento geográfico desigual no Brasil. REDES, Santa Cruz do Sul, v. 14, n. 1, p. 62-81, jan./abr. 2009.

VECHIATO, Fernando Luiz. Repositório digital como ambiente de inclusão digital e social para usuários idosos. 2010. 185 f. Dissertação (Mestrado em Ciência da Informação)-Universidade Estadual Paulista, Marília, SP, 2010.

VERONA, Silvana Marinaro et al. Percepção do idoso em relação à internet. Periódicos Eletrônicos em Psicologia, Ribeirão Preto, v. 14, n. 2, p. 1-9, dez. 2006.

VIEIRA, Maristela Compagnoni. O velho e o novo: caminhos para entender a relação dos idosos com as tecnologias digitais. 2011. 184 f. Dissertação (Mestrado em Educação)-Universidade Federal do Rio Grande do Sul, Porto Alegre, 2011.

Data de submissão: 09/06/2016

Data de aprovação: 06/05/2017 
